\title{
Myocardial Fibrosis as an Early Manifestation of Hypertrophic Cardiomyopathy
}

\author{
Carolyn Y. Ho, M.D., Begoña López, Ph.D., Otavio R. Coelho-Filho, M.D., \\ Neal K. Lakdawala, M.D., Allison L. Cirino, M.S., C.G.C., Petr Jarolim, M.D., Ph.D., \\ Raymond Kwong, M.D., Arantxa González, Ph.D., Steven D. Colan, M.D., \\ J.G. Seidman, Ph.D., Javier Díez, M.D., Ph.D., and Christine E. Seidman, M.D.
}

From the Cardiovascular Division (C.Y.H., O.R.C.-F., N.K.L., A.L.C., R.K., J.G.S., C.E.S.) and the Department of Pathology (P.J.), Brigham and Women's Hospital; the Department of Cardiology, Children's Hospital Boston (S.D.C.); and the Department of Genetics, Harvard Medical School (J.G.S., C.E.S.) - all in Boston; the Division of Cardiovascular Sciences, Center of Applied Medical Research and University Clinic, University of Navarra, Pamplona, Spain (B.L., A.G., J.D.); and the Howard Hughes Medical Institute, Chevy Chase, MD (C.E.S.). Address reprint requests to Dr. Ho at the Cardiovascular Division, Brigham and Women's Hospital, 75 Francis St., Boston, MA 02115, or atcho@partners.org.

N Engl J Med 2010;363:552-63.

Copyright (c) 2010 Massachusetts Medical Society.

\section{ABSTRACT}

\section{BACKGROUND}

Myocardial fibrosis is a hallmark of hypertrophic cardiomyopathy and a proposed substrate for arrhythmias and heart failure. In animal models, profibrotic genetic pathways are activated early, before hypertrophic remodeling. Data showing early profibrotic responses to sarcomere-gene mutations in patients with hypertrophic cardiomyopathy are lacking.

\section{METHODS}

We used echocardiography, cardiac magnetic resonance imaging (MRI), and serum biomarkers of collagen metabolism, hemodynamic stress, and myocardial injury to evaluate subjects with hypertrophic cardiomyopathy and a confirmed genotype.

\section{RESULTS}

The study involved 38 subjects with pathogenic sarcomere mutations and overt hypertrophic cardiomyopathy, 39 subjects with mutations but no left ventricular hypertrophy, and 30 controls who did not have mutations. Levels of serum C-terminal propeptide of type I procollagen (PICP) were significantly higher in mutation carriers without left ventricular hypertrophy and in subjects with overt hypertrophic cardiomyopathy than in controls (31\% and $69 \%$ higher, respectively; $\mathrm{P}<0.001$ ). The ratio of PICP to C-terminal telopeptide of type I collagen was increased only in subjects with overt hypertrophic cardiomyopathy, suggesting that collagen synthesis exceeds degradation. Cardiac MRI studies showed late gadolinium enhancement, indicating myocardial fibrosis, in $71 \%$ of subjects with overt hypertrophic cardiomyopathy but in none of the mutation carriers without left ventricular hypertrophy.

\section{CONCLUSIONS}

Elevated levels of serum PICP indicated increased myocardial collagen synthesis in sarcomere-mutation carriers without overt disease. This profibrotic state preceded the development of left ventricular hypertrophy or fibrosis visible on MRI. (Funded by the National Institutes of Health and others.) 
$\mathrm{H}$ YPERTROPHIC CARDIOMYOPATHY IS caused by mutations in genes encoding sarcomere proteins. ${ }^{1,2}$ With a prevalence of approximately 1 case per 500 persons in the general population, hypertrophic cardiomyopathy is the most common monogenic cardiac disorder. ${ }^{3}$ The clinical diagnosis depends on the identification of unexplained left ventricular hypertrophy, but this finding is present only in persons with established disease and is typically absent in childhood. ${ }^{4}$ In contrast, genetic diagnosis identifies pathogenic sarcomere mutations in persons at any age, including mutation carriers with overt hypertrophic cardiomyopathy and mutation carriers without hypertrophy who are at high risk for the development of disease. Studying such mutation carriers may provide insight into the pathophysiology of hypertrophic cardiomyopathy by revealing subtle, early manifestations of sarcomere mutations that precede the obvious pathologic remodeling of overt disease. For example, left ventricular relaxation is impaired in mutation carriers without left ventricular hypertrophy, indicating that sarcomere mutations directly affect diastolic function..$^{5-7}$

Myocardial fibrosis, a hallmark of hypertrophic cardiomyopathy, is thought to contribute to sudden cardiac death, ventricular tachyarrhythmias, left ventricular dysfunction, and heart failure. ${ }^{8-12}$ Histologic evaluation universally reveals increased interstitial and focal myocardial fibrosis in overt disease. In most patients with overt hypertrophic cardiomyopathy, dense focal fibrosis can also be visualized noninvasively with the use of gadolinium-enhanced cardiac magnetic resonance imaging (MRI). ${ }^{10,13-18}$ The trigger for increased myocardial fibrosis in hypertrophic cardiomyopathy remains unclear, but it has been attributed both to premature myocyte death caused by stresses imposed directly by sarcomere mutations ${ }^{19-22}$ and to later pathologic changes, including intracavitary obstruction, small-vessel disease, and ischemia. ${ }^{9,12,23}$ Animal models of hypertrophic cardiomyopathy that recapitulate human disease ${ }^{24}$ have recently shed light on the earliest cellular and molecular responses to sarcomere-gene mutations. ${ }^{25}$ Cardiac transcriptional profiling in young mice in which hypertrophy has not yet developed shows activation of pathways involved in fibrosis and collagen deposition. ${ }^{25}$ These studies indicate that a profibrotic milieu is present early in hearts with hypertro- phic cardiomyopathy, even when cardiac histologic findings are normal.

Biomarkers of collagen synthesis and degradation reflect collagen metabolism. The $\mathrm{C}$-terminal propeptide of type I procollagen (PICP) is released in a 1:1 ratio during the synthesis of type I collagen from its precursor, procollagen type I, ${ }^{26}$ and serum levels reliably reflect myocardial type I collagen synthesis. ${ }^{27}$ Increased serum PICP levels correlate with adverse outcomes in hypertension, heart failure, and myocardial infarction. ${ }^{28-30}$

There have been few studies of these biomarkers in hypertrophic cardiomyopathy, although preliminary studies of nongenotyped patients with overt disease suggest that collagen turnover is increased. ${ }^{31-34}$ Data regarding collagen metabolism in patients with early disease are lacking. We therefore measured serum biomarkers of collagen metabolism to assess profibrotic processes in a genotyped population with hypertrophic cardiomyopathy, comparing mutation carriers in whom left ventricular hypertrophy had not yet developed with those who had overt disease and with mutation-negative normal controls.

\section{METHODS}

\section{STUDY POPULATION}

Genotyped patients with hypertrophic cardiomyopathy and their relatives, identified through research studies or clinical evaluation, were recruited and assigned to one of three groups. Mutation carriers with a left ventricular wall thickness of $12 \mathrm{~mm}$ or greater (in adults) or a $\mathrm{z}$ score of 2 or more (in children) ${ }^{35}$ were classified as having overt hypertrophic cardiomyopathy. Healthy mutation carriers who did not meet these criteria were classified as mutation carriers without left ventricular hypertrophy. The criteria we used are more rigorous than those used clinically to diagnose hypertrophic cardiomyopathy. ${ }^{36}$ They were chosen to avoid the inclusion of subjects with borderline left ventricular hypertrophy and potentially emerging or mild cardiomyopathy in the group of mutation carriers without left ventricular hypertrophy. Control subjects were healthy, mutation-negative relatives at ages similar to those of mutation carriers without left ventricular hypertrophy.

The study protocol was approved by the institutional review boards of Brigham and Women's Hospital and Children's Hospital Boston, and the 
study was conducted in accordance with the protocol. Written informed consent was obtained from all participants or their parents or legal guardians.

Subjects with systemic hypertension (defined by systolic blood pressure $\geq 140 \mathrm{~mm} \mathrm{Hg}$, diastolic blood pressure $\geq 90 \mathrm{~mm} \mathrm{Hg}$, or use of antihypertensive medication), coronary artery disease, valvular heart disease, previous septal myectomy, alcohol septal ablation, electronic ventricular pacing, or atrial fibrillation were excluded. Subjects with other conditions that might influence collagen metabolism (e.g., surgery or trauma within the previous 6 months, known fibrotic or inflammatory disease, or cancer) were also excluded.

\section{ECHOCARDIOGRAPHY}

Transthoracic echocardiograms were obtained with the use of a Vivid 7 ultrasonography system (General Electric Medical Systems). The average of three cardiac cycles was used for measurements of cardiac dimensions, mitral inflow patterns, and the left ventricular ejection fraction (calculated according to Simpson's method). ${ }^{37}$ Myocardial velocities during systole and early diastole were measured by means of tissue Doppler imaging at the lateral, septal, anterior, and inferior aspects of the mitral annulus in the apical four-chamber and two-chamber views. Global values of systolic and early diastolic myocardial velocities were calculated as the average of these four measurements to represent myocardial contraction and relaxation, respectively. The ratio of early mitral inflow velocity to global early diastolic velocity approximately represented left ventricular end-diastolic pressure. ${ }^{38}$ Measures of left ventricular stiffness were evaluated, including E-wave deceleration time, end-systolic elastance ([0.9 $\times$ systolic blood pressure $] \div$ left ventricular end-systolic volume), and stiffness $(70 \div$ [E-wave deceleration time -20$])^{2}$ or (E-wave deceleration time $\div$ early mitral inflow velocity). ${ }^{39,40}$ Images were analyzed by two observers who were unaware of the subjects' clinical and genetic status.

\section{CARDIAC MR}

Cardiac MRI studies were performed in a subgroup of subjects with overt hypertrophic cardiomyopathy and in mutation carriers without left ventricular hypertrophy, with the use of a 1.5-T cardiac MRI system (HDX Excite II, General Electric) or a 3.0-T system (Magnetom Trio, Siemens).
Steady-state free precession of cine images was used to quantify left ventricular function (shortaxis stack, slice thickness of $8 \mathrm{~mm}$ ) and myocardial mass by means of standard criteria. ${ }^{41} \mathrm{Seg}$ mented inversion-recovery fast gradient-echo imaging was used to assess late gadolinium enhancement 10 minutes after the administration of gadolinium diethylenetriamine pentaacetic acid at a dose of $0.15 \mathrm{mmol}$ per kilogram of body weight. The extent of late gadolinium enhancement was quantified by planimetric assessment of all short-axis slices for total volume (the sum of the areas measured, in grams) and as a proportion of the total left ventricular mass (the percentage of late gadolinium enhancement). Late gadolinium enhancement was defined as 2 standard deviations above the mean signal intensity of the distant myocardium. ${ }^{42}$ Images were analyzed offline with the use of QMass MR software (Medis) by two observers who were unaware of the subjects' clinical and genetic information.

\section{MEASUREMENT OF SERUM BIOMARKERS}

Blood samples (serum and plasma) were obtained at the time of cardiac imaging, processed within 60 minutes after phlebotomy, and stored at $-80^{\circ} \mathrm{C}$ before analysis of PICP, matrix metalloproteinase 1 (MMP-1), tissue inhibitor of metalloproteinase 1 (TIMP-1), C-terminal telopeptide of type I collagen (CITP), osteopontin, bone-specific alkaline phosphatase, N-terminal propeptide of B-type natriuretic peptide, B-type natriuretic peptide, and cardiac troponin I. All assays were performed with the use of commercially available reagents (see the Methods section in the Supplementary Appendix, available with the full text of this article at NEJM.org) by investigators who were unaware of the subjects' clinical and genetic status.

\section{STATISTICAL ANALYSIS}

To test for differences among the three groups and between subjects with $\beta$-myosin heavy chain (MYH7) mutations and those with cardiac myosin-binding protein $\mathrm{C}$ (MYBPC3) mutations, analysis of variance and logistic regression were performed with clustering to adjust for family relationships, assuming an exchangeable correlation structure. Age-dependent mitral inflow, myocardial velocities measured by tissue Doppler imaging, and biomarker patterns were adjusted for age; biomarkers were also adjusted for sex. Values are expressed as adjusted means \pm SE. 
Bonferroni-corrected $\mathrm{P}$ values of less than 0.017 were considered to indicate statistical significance for multiple comparisons across the three groups. Pearson's correlation was used to evaluate associations between continuous measures. Analyses were performed with the use of SAS software, version 9.1 (SAS Institute).

\section{RESULTS}

\section{CLINICAL CHARACTERISTICS}

Study procedures were performed in 107 subjects. Forty-two different pathogenic mutations ${ }^{7}(37$ MYH7, 31 MYBPC3, 9 cardiac troponin T [TNNT2], and $1 \alpha$-tropomyosin [TPM1]) were identified in 47 families with hypertrophic cardiomyopathy (Table 1 in the Supplementary Appendix).

On the basis of clinical evaluations and genotypes, 38 subjects were classified as mutation carriers with overt hypertrophic cardiomyopathy, 39 as mutation carriers without left ventricular hypertrophy, and 30 as having neither mutations nor hypertrophic cardiomyopathy (control subjects) (Table 1). Mutation carriers without left ventricular hypertrophy and control subjects were of similar age, were asymptomatic, were not receiving cardiac medications, and had normal cardiac dimensions. These subjects had slightly lower blood pressure than controls, and they also had a higher left ventricular ejection fraction and a lower global early diastolic myocardial velocity, as reported previously for such carriers. ${ }^{6,7}$

Subjects with overt hypertrophic cardiomyopathy had a significantly greater left ventricular wall thickness $(76 \%$ with asymmetric septal hypertrophy), smaller left ventricular cavity, and larger left atrial diameter than mutation carriers without left ventricular hypertrophy and control subjects. Sixteen percent had intracavitary obstruction at rest (peak gradient, $\geq 30 \mathrm{~mm} \mathrm{Hg}$ ). Symptoms were generally mild in subjects with overt hypertrophic cardiomyopathy $(74 \%$ had New York Heart Association [NYHA] class I status and $21 \%$ had NYHA class II status). Three subjects had NYHA class III symptoms, including two subjects with end-stage hypertrophic cardiomyopathy, defined by a reduced left ventricular ejection fraction $(<50 \%)$. Sixty-eight percent of the subjects with overt hypertrophic cardiomyopathy were receiving cardioactive medications, including eight subjects receiving angiotensin- converting-enzyme (ACE) inhibitors, angiotensinreceptor blockers (ARBs), or spironolactone.

\section{IMAGING OF FIBROSIS WITH CARDIAC MRI}

Cardiac MRI studies with the administration of gadolinium were performed in 28 subjects with overt hypertrophic cardiomyopathy and $32 \mathrm{mu}-$ tation carriers without left ventricular hypertrophy. Studies were not performed in 9 subjects with overt hypertrophic cardiomyopathy who had implantable cardioverter-defibrillators and in 5 mutation-positive children without left ventricular hypertrophy whose parents declined. In 3 subjects, gadolinium was not administered. Late gadolinium enhancement was present in $71 \%$ of subjects with overt hypertrophic cardiomyopathy but in none of the mutation carriers without left ventricular hypertrophy (Fig. 1). The extent of late gadolinium enhancement in subjects with overt hypertrophic cardiomyopathy ranged from 0 to $55 \%$ of the total left ventricular mass (mean, $14.7 \pm 16.9 \%)$. There were significant correlations between the extent of late gadolinium enhancement in these subjects and the left ventricular mass, ejection fraction, global early diastolic myocardial velocity, and ratio of early mitral inflow velocity to early diastolic velocity (Tables 2 and 3 in the Supplementary Appendix). There were no significant correlations between late gadolinium enhancement and levels of cardiac troponin I, B-type natriuretic peptide, PICP, CITP, MMP-1, or TIMP-1; age; left ventricular hypertrophy; size of the left atrium; or E-wave deceleration time.

\section{INCREASED MYOCARDIAL COLLAGEN SYNTHESIS}

Analyses of serum biomarkers were performed after exclusion of the eight subjects with overt hypertrophic cardiomyopathy who were receiving ACE inhibitors, ARBs, or spironolactone (including the two subjects with end-stage hypertrophic cardiomyopathy), since these factors may influence collagen metabolism. As compared with PICP levels in controls $(82.16 \pm 3.03 \mu \mathrm{g}$ per liter), the PICP levels were significantly elevated in mutation carriers without left ventricular hypertrophy $(107.73 \pm 4.65 \mu \mathrm{g}$ per liter, a $31 \%$ elevation) and in subjects with overt hypertrophic cardiomyopathy $(138.70 \pm 11.63 \mu \mathrm{g}$ per liter, a $69 \%$ elevation; $\mathrm{P}<0.001$ for both comparisons with controls) (Table 2 and Fig. 1 in the Supplementary Appendix). PICP was the only biomarker that dif- 


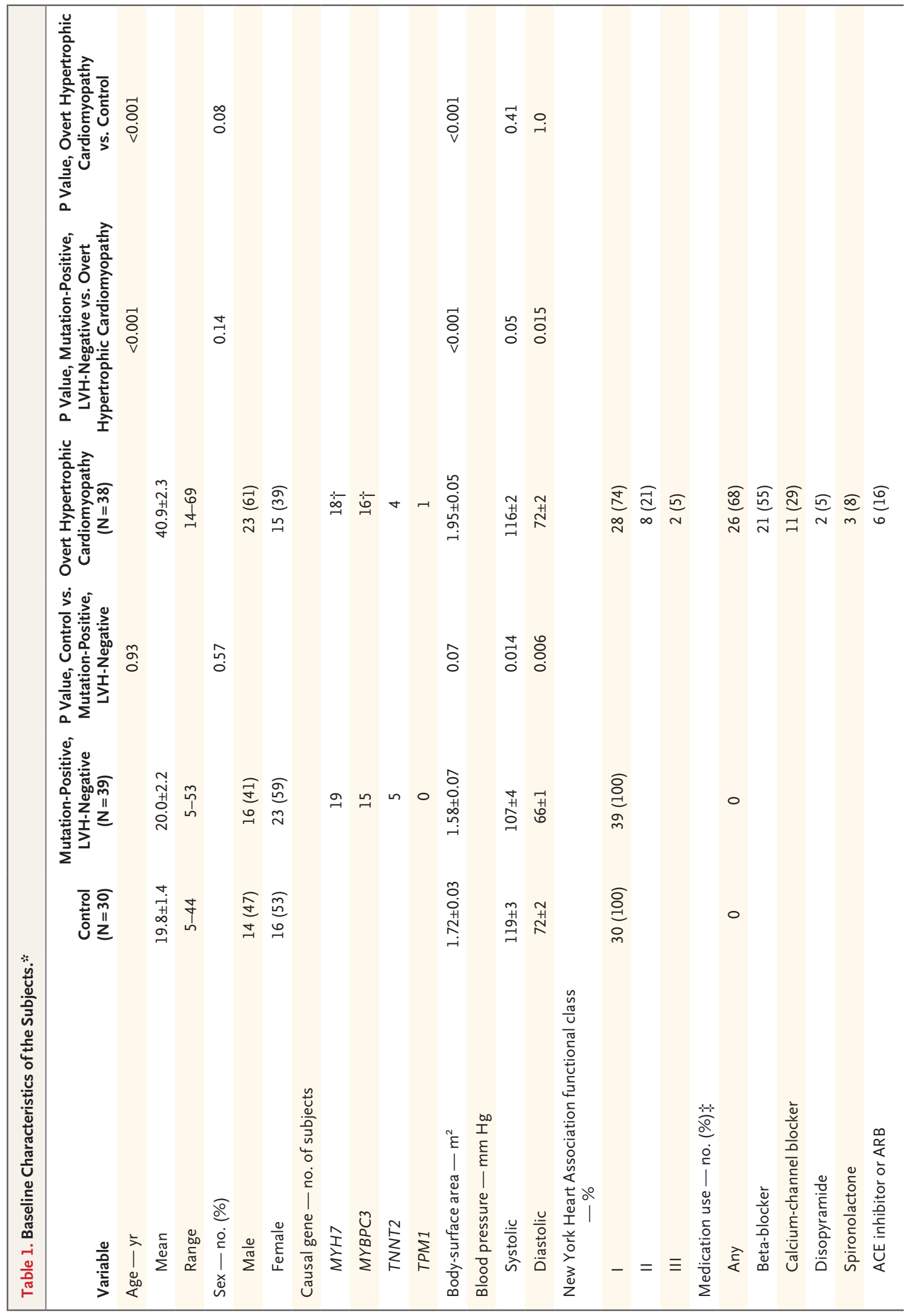




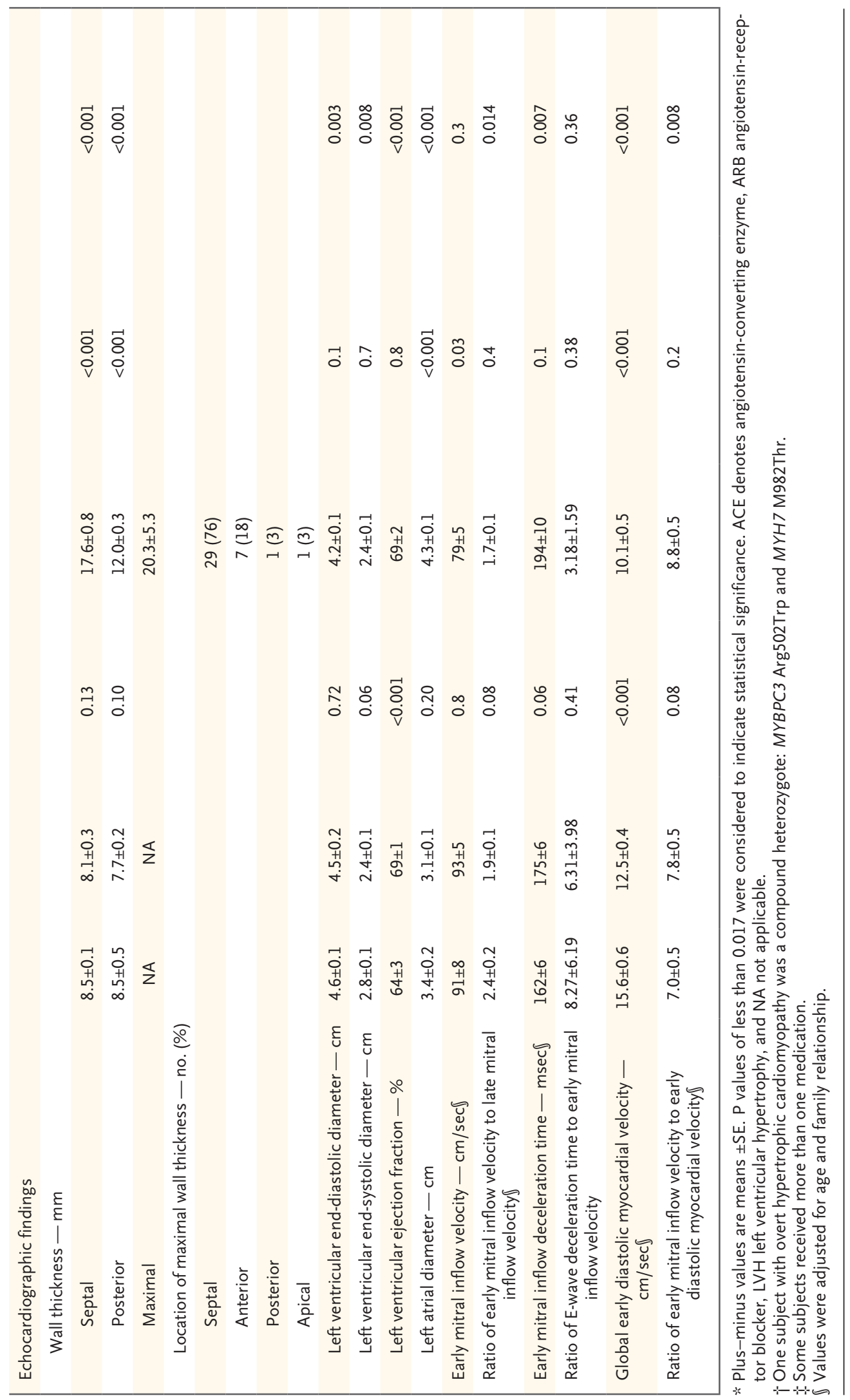




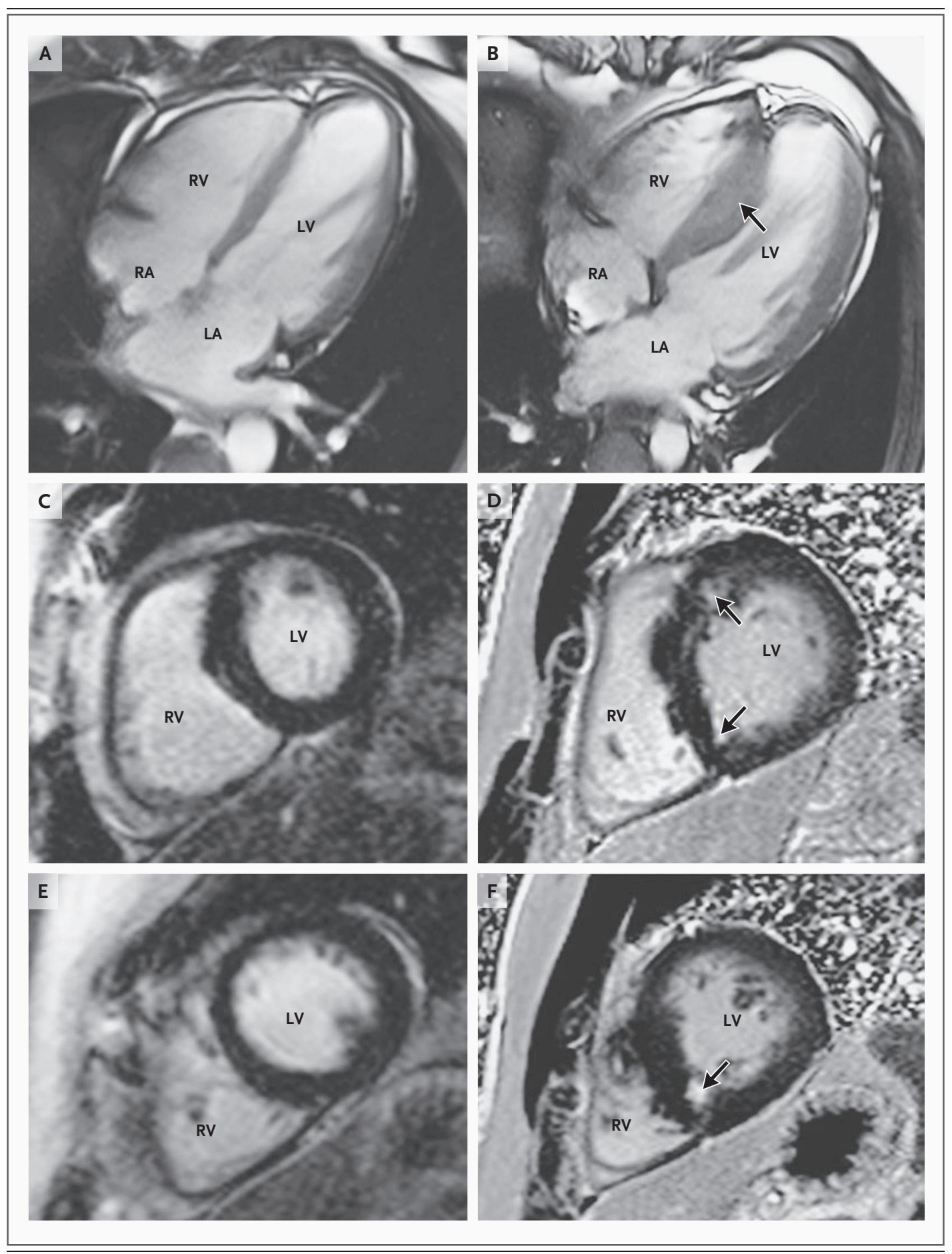

fered between mutation carriers and noncarriers (Table 2). Among all family members with a normal left ventricular wall thickness, the odds of carrying a mutation increased by $50 \%$ for every increase in PICP of $10 \mu \mathrm{g}$ per liter, after adjustment for age, sex, early diastolic myocardial ve- locity, and left ventricular ejection fraction (odds ratio, 1.50; 95\% confidence interval [CI], 1.18 to 1.91; $\mathrm{P}=0.001)$.

Type I collagen is a major component of bone; therefore, PICP levels may reflect bone formation, particularly in growing children and ado- 
Figure 1 (facing page). Gadolinium-Enhanced Cardiac MRI Scans in MYH7 Mutation Carriers with and Those without Overt Hypertrophic Cardiomyopathy.

Panels A, C, and E show no morphologic abnormalities suggestive of hypertrophic cardiomyopathy in a carrier of a myosin heavy-chain missense mutation. Left ventricular hypertrophy and late gadolinium enhancement are absent. Panels $B, D$, and $F$ show typical findings of hypertrophic cardiomyopathy in a relative of the subject in Panels A, C, and E with overt disease caused by the same myosin heavy-chain mutation. These findings in clude a markedly thickened septum (Panel B, arrow) associated with areas of heterogeneous late gadolinium enhancement, which affects $17 \%$ of the total left ventricular mass and involves the anteroseptum (Panel D, arrows) and the inferoseptum (Panels D and F, arrows). LA denotes left atrium, LV left ventricle, RA right atrium, and $\mathrm{RV}$ right ventricle.

lescents. ${ }^{43}$ To minimize such confounding, we measured levels of bone-specific alkaline phosphatase (a specific marker of bone metabolic activity) ${ }^{43}$ and the ratio of PICP to bone-specific alkaline phosphatase to adjust PICP levels for the influence of bone collagen turnover. As compared with levels of bone-specific alkaline phosphatase in controls, levels were $17 \%$ lower in mutation carriers without left ventricular hypertrophy $(\mathrm{P}=0.003)$ and were $20 \%$ lower in subjects with overt hypertrophic cardiomyopathy $(\mathrm{P}=0.006)$ (Fig. 1B in the Supplementary Appendix). The ratio of PICP to bone-specific alkaline phosphatase was significantly higher both in mutation carriers without left ventricular hypertrophy and in subjects with overt hypertrophic cardiomyopathy than in controls $(77 \%$ higher in mutation carriers but no left ventricular hypertrophy, $\mathrm{P}<0.001$ ) (Fig. 1C in the Supplementary Appendix). These results suggest that elevated PICP levels in sarcomere-mutation carriers reflect increased myocardial collagen synthesis rather than increased bone metabolic activity.

No significant differences in markers of collagen degradation (MMP-1, TIMP-1, and CITP) were identified in isolation. The PICP:CITP ratio reflects the balance between collagen synthesis and degradation. ${ }^{44}$ As compared with the PICP:CITP ratio in control subjects, the ratio was unchanged in mutation carriers without left ventricular hypertrophy but significantly higher in subjects with overt hypertrophic cardiomyopathy, suggesting that collagen synthesis exceeds degradation in established disease.

\section{CLINICAL AND GENETIC ASSOCIATIONS} WITH INCREASED PICP LEVELS

No significant correlations were identified between increased PICP levels and levels of B-type natriuretic peptide, N-terminal propeptide of B-type natriuretic peptide, or cardiac troponin I; the degree of left ventricular hypertrophy; left ventricular mass; left atrial size; left ventricular systolic or diastolic function; echocardiographic measurements of left ventricular stiffness; or late gadolinium enhancement on cardiac MRI in any cohort. Among mutation carriers without left ventricular hypertrophy, there were also no significant differences in mean PICP levels between subjects with and those without electrocardiographic (ECG) abnormalities, including Q waves (which were present in 36\% of mutation carriers without left ventricular hypertrophy).

We compared subjects who had MYH7 mutations and those who had MYBPC3 mutations to assess potential genotype-phenotype correlations in the two most common genetic subtypes of hypertrophic cardiomyopathy (Table 3). PICP levels were $14 \%$ higher in subjects without left ventricular hypertrophy who had MYH7 mutations than in subjects without left ventricular hypertrophy who had MYBPC3 mutations $(\mathrm{P}=0.014)$. Global early myocardial diastolic velocities were $15 \%$ lower in subjects without left ventricular hypertrophy who had MYH7 mutations $(\mathrm{P}=0.002)$ than in those who had MYBPC3 mutations, in whom the velocities were essentially normal. Among subjects with overt hypertrophic cardiomyopathy, PICP levels did not differ significantly between those who had MYH7 mutations and those who had MYBPC3 mutations, although early myocardial diastolic velocities were lower in subjects with MYH7 mutations.

\section{DISCUSSION}

We present data showing increased myocardial type I collagen synthesis in both early and established hypertrophic cardiomyopathy. Elevated serum PICP levels in mutation carriers with normal cardiac morphologic features constitute a potentially useful phenotype for sarcomere mutations and a serologic marker of genetic risk that can be detected before clinical diagnosis. These observations offer insights into the pathophysiology of hypertrophic cardiomyopathy, suggesting that the stimulus for myocardial 


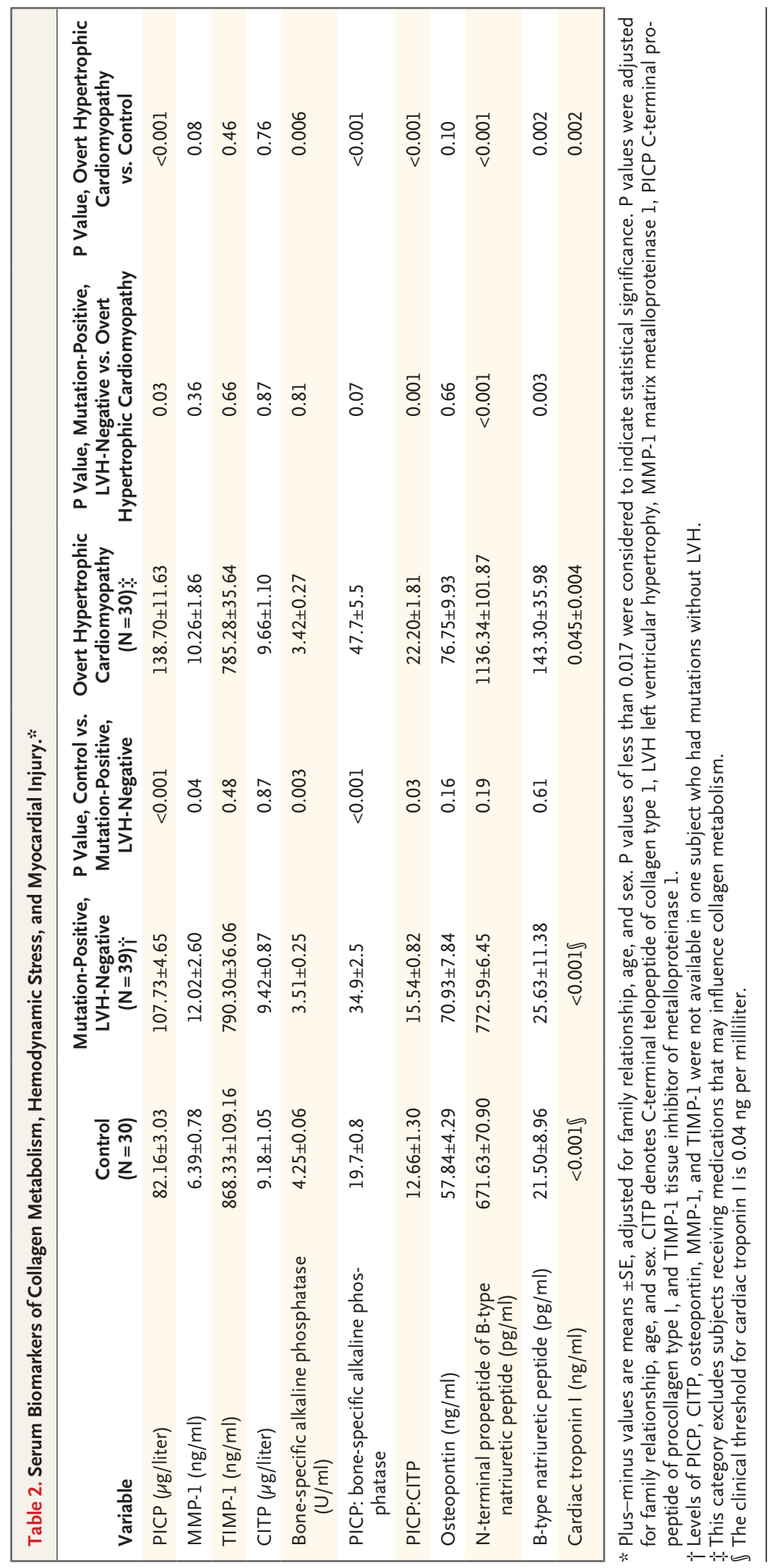


fibrosis is an early manifestation of sarcomeregene mutations.

In contrast to collagen synthesis, biomarkers of collagen degradation in isolation were not informative regarding genotype or clinical status. However, the PICP:CITP ratio, an index of the dynamic equilibrium between collagen type I synthesis and degradation, ${ }^{44}$ was unchanged in mutation carriers without left ventricular hypertrophy but increased in subjects with overt disease. We propose that sarcomere mutations trigger an early increase in collagen synthesis that is initially balanced by degradation, thereby limiting fibrogenesis. In overt hypertrophic cardiomyopathy, synthesis exceeds degradation, resulting in frank myocardial fibrosis. Although our study was not longitudinal, these results are consistent with the natural history of hypertrophic cardiomyopathy and suggest that collagen accumulation increases as disease develops. Indeed, these findings may partially explain why late gadolinium enhancement on cardiac MRI was present in the majority of our subjects with overt hypertrophic cardiomyopathy, as observed in other studies, ${ }^{10,13,14,16-18}$ but absent in the mutation carriers without left ventricular hypertrophy, despite elevated PICP levels.

Increased myocardial fibrosis is a hallmark of overt hypertrophic cardiomyopathy and is frequently interpreted as a secondary response to the pathophysiological remodeling of long-standing disease, including ischemia, obstruction, and microvascular abnormalities.9,12,23 This interpretation is challenged by studies showing early activation of profibrotic genetic pathways in a mouse model of hypertrophic cardiomyopathy. In young mice with a myosin heavy-chain mutation, comprehensive transcriptional profiling revealed increased expression of genes that drive extracellular-matrix formation before fibrosis or left ventricular hypertrophy develops. ${ }^{25}$ RNA levels of connective-tissue growth factor, periostin, transforming growth factor $\beta 1$ and $\beta 2$, fibronectin, and type I collagen were increased despite normal cardiac histologic findings.

Our data translate these findings from mouse models to human disease, providing further evidence that fibrosis is a fundamental, early consequence of sarcomere mutations. On the basis of the elevated PICP levels in mutation carriers without left ventricular hypertrophy, we propose that increased collagen synthesis contributes to

\begin{tabular}{|c|c|c|c|}
\hline Variable & MYH7 & MYBPC3 & P Value \\
\hline \multicolumn{4}{|l|}{ Overt hypertrophic cardiomyopathy } \\
\hline No. of subjects & 17 & 16 & \\
\hline Age $-y r$ & $36.9 \pm 3.5$ & $44.7 \pm 2.6$ & 0.07 \\
\hline Sex - no. (\%) & & & 0.64 \\
\hline Male & $11(65)$ & $9(56)$ & \\
\hline Female & $6(35)$ & $7(44)$ & \\
\hline $\begin{array}{l}\text { Maximal left ventricular wall thickness } \\
\qquad-\mathrm{mm}\end{array}$ & $20.5 \pm 1.35$ & $21.2 \pm 1.4$ & 0.72 \\
\hline $\begin{array}{l}\text { Left ventricular mass on cardiac } \\
\text { MRI — g }\end{array}$ & $186.8 \pm 19.3$ & $147.2 \pm 9.8$ & 0.07 \\
\hline $\begin{array}{l}\text { Global early diastolic velocity - } \\
\mathrm{cm} / \mathrm{sec}\end{array}$ & $6.9 \pm 0.5$ & $9.3 \pm 0.4$ & $<0.001$ \\
\hline Left ventricular ejection fraction — \% & $70 \pm 2$ & $69 \pm 2$ & 0.79 \\
\hline $\begin{array}{l}\text { Late gadolinium enhancement } \\
\text { volume }-\mathrm{g}\end{array}$ & $30.8 \pm 10.3$ & $18.8 \pm 7.0$ & 0.35 \\
\hline $\begin{array}{c}\text { Late gadolinium enhancement - \% } \\
\text { of total left ventricular mass }\end{array}$ & $18.9 \pm 6.6$ & $11.7 \pm 4.2$ & 0.39 \\
\hline $\mathrm{PICP}-\mu \mathrm{g} /$ liter & $123.01 \pm 9.28$ & $124.16 \pm 1.07$ & 0.91 \\
\hline MMP-1 - ng/ml & $8.37 \pm 2.23$ & $8.70 \pm 2.18$ & 0.92 \\
\hline \multicolumn{4}{|l|}{ Mutation-positive, LVH-negative } \\
\hline No. of subjects & 19 & 15 & \\
\hline Age $-y r$ & $18.2 \pm 3.4$ & $21.0 \pm 3.6$ & 0.57 \\
\hline Sex - no. (\%) & & & 0.32 \\
\hline Male & $8(42)$ & $4(27)$ & \\
\hline Female & $11(58)$ & $11(73)$ & \\
\hline $\begin{array}{l}\text { Global early diastolic velocity }-\mathrm{cm} / \\
\text { sec }\end{array}$ & $12.9 \pm 0.5$ & $15.1 \pm 0.5$ & 0.002 \\
\hline Left ventricular ejection fraction — \% & $71 \pm 1$ & $69 \pm 2$ & 0.32 \\
\hline $\mathrm{PICP}-\mu \mathrm{g} /$ liter & $123.51 \pm 1.20$ & $108.10 \pm 5.06$ & 0.014 \\
\hline MMP-1 — ng/ml & $11.26 \pm 4.29$ & $15.47 \pm 4.56$ & 0.51 \\
\hline
\end{tabular}

* Plus-minus values are means $\pm \mathrm{SE}$, adjusted for age. P values of less than 0.05 were considered to indicate statistical significance; $P$ values were adjusted for family relationship and age. LVH denotes left ventricular hypertrophy, MMP-I matrix metalloproteinase 1, MRI magnetic resonance imaging, MYBPC3 cardiac myosin-binding protein C, MYH7 cardiac $\beta$-myosin heavy chain, and PICP C-terminal propeptide of procollagen type I.

the emergence of the pathophysiological changes that characterize overt hypertrophic cardiomyopathy. Analysis of additional biomarkers (collagen type III-derived peptides, other matrix metalloproteinases, and TIMPs) may provide a more comprehensive picture of how sarcomere mutations affect the complex and dynamic nature of fibrillar collagen metabolism.

We could not identify significant correlations between biomarker levels and other clinical variables, including late gadolinium enhancement 
on cardiac MRI and ECG abnormalities. These results are not surprising, given the limited resolution of late gadolinium enhancement for the detection of focal myocardial fibrosis (theoretical detection limit, approximately $0.2 \mathrm{~g}$ of scar; a clinical detection limit of approximately $2.0 \mathrm{~g}$ in infarct studies ${ }^{45,46}$ ) and estimates of the amount of scar (approximately 3\% of left ventricular mass) needed to produce changes visible on ECG studies. ${ }^{47}$ Moreover, diffuse interstitial fibrosis is common in hypertrophic cardiomyopathy but cannot be visualized by means of cardiac MRI. In contrast, immunoassays detect micrograms of circulating PICP. Serum biomarkers consequently provide a more sensitive index of extracellularmatrix remodeling and may reflect subtle changes in myocardial composition and biochemical features that are not detectable by means of noninvasive cardiac imaging. Notably, we observed that MYH7 mutation carriers without left ventricular hypertrophy had both higher PICP levels (indicating increased collagen synthesis) and lower early diastolic levels (indicating more impaired relaxation) than MYBPC3 mutation carriers. Previous studies suggest that mutations in MYH7 result in an earlier onset of overt hypertrophic cardiomyopathy than do MYBPC3 mutations. ${ }^{48,49}$ Our data provide a potential mechanism for this genotype-phenotype correlation - namely, that MYH7 mutations, as compared with MYBPC3 mutations, trigger earlier extracellular-matrix remodeling, more extensive remodeling, or both.

The identification of increased myocardial col- lagen synthesis in mutation carriers without left ventricular hypertrophy, as with diastolic dysfunction in this population, ${ }^{5-7}$ shows that sarcomere-gene mutations have a considerable effect on the heart before the onset of hypertrophy. The detection of a profibrotic myocardial milieu in mutation carriers without left ventricular hypertrophy has intriguing clinical implications. Increased serologic markers of collagen synthesis may identify persons at risk for arrhythmias, sudden death, or heart failure. If so, monitoring levels of these markers may guide new strategies to attenuate disease development or adverse outcomes in hypertrophic cardiomyopathy. We suggest that incorporating genetic testing to identify at-risk mutation carriers, defining features of early disease, and developing therapies to mitigate fibrosis will foster vital new opportunities to change the natural history of hypertrophic cardiomyopathy.

Supported by grants from the National Institutes of Health (to Drs. Ho, J.G. Seidman, and C.E. Seidman), the Arthur L. Lenahan Sr. Family Foundation (to Dr. Ho), the GlaxoSmithKline Research and Education Foundation for Cardiovascular Disease (to Dr. Ho), the Howard Hughes Medical Institute (to Dr. C.E. Seidman), and the Foundation for Applied Medical Research and Unión Temporal de Empresas project Centro de Investigación Médica Aplicada, the Red Temática de Investigación Cooperativa en Enfermedades Cardiovasculares from the Institute of Health Carlos III, Ministry of Science and Innovation, and the European Union InGenious HyperCare (all to Drs. López, González, and Díez).

Disclosure forms provided by the authors are available with the full text of this article at NEJM.org.

We thank Sonia Martínez and Lucy Panov for technical assistance in the measurement of serum biomarkers.
REFERENCES

1. Seidman JG, Seidman C. The genetic basis for cardiomyopathy: from mutation identification to mechanistic paradigms. Cell 2001;104:557-67.

2. Richard P, Villard E, Charron P, Isnard $\mathrm{R}$. The genetic bases of cardiomyopathies. J Am Coll Cardiol 2006;48:79-89.

3. Maron BJ, Gardin JM, Flack JM, Gidding SS, Kurosaki TT, Bild DE. Prevalence of hypertrophic cardiomyopathy in a general population of young adults: echocardiographic analysis of 4111 subjects in the CARDIA study. Circulation 1995;92: 785-9.

4. Maron BJ, Seidman JG, Seidman CE. Proposal for contemporary screening strategies in families with hypertrophic cardiomyopathy. J Am Coll Cardiol 2004; 44:2125-32

5. Nagueh SF, Bachinski LL, Meyer D, et al. Tissue Doppler imaging consistently detects myocardial abnormalities in patients with hypertrophic cardiomyopathy and provides a novel means for an early diagnosis before and independently of hypertrophy. Circulation 2001;104:128-30. 6. Ho CY, Sweitzer NK, McDonough B, et al. Assessment of diastolic function with Doppler tissue imaging to predict genotype in preclinical hypertrophic cardiomyopathy. Circulation 2002;105:2992-7. 7. Ho CY, Carlsen C, Thune JJ, et al. Echocardiographic strain imaging to assess early and late consequences of sarcomere mutations in hypertrophic cardiomyopathy. Circ Cardiovasc Genet 2009; 2:314-21

8. Basso C, Thiene G, Corrado D, Buja G, Melacini P, Nava A. Hypertrophic cardiomyopathy and sudden death in the young: pathologic evidence of myocardial ischemia. Hum Pathol 2000;31:988-98.
9. Shirani J, Pick R, Roberts WC, Maron BJ. Morphology and significance of the left ventricular collagen network in young patients with hypertrophic cardiomyopathy and sudden cardiac death. J Am Coll Cardiol 2000;35:36-44.

10. Choudhury L, Mahrholdt H, Wagner A, et al. Myocardial scarring in asymptomatic or mildly symptomatic patients with hypertrophic cardiomyopathy. J Am Coll Cardiol 2002;40:2156-64.

11. Varnava AM, Elliott PM, Mahon N, Davies MJ, McKenna WJ. Relation between myocyte disarray and outcome in hypertrophic cardiomyopathy. Am J Cardiol 2001;88:275-9.

12. Varnava AM, Elliott PM, Sharma S, McKenna WJ, Davies MJ. Hypertrophic cardiomyopathy: the interrelation of disarray, fibrosis, and small vessel disease. Heart 2000;84:476-82 
13. Moon JC, McKenna WJ, McCrohon JA, Elliott PM, Smith GC, Pennell DJ. Toward clinical risk assessment in hypertrophic cardiomyopathy with gadolinium cardiovascular magnetic resonance. J Am Coll Cardiol 2003;41:1561-7.

14. Adabag AS, Maron BJ, Appelbaum E, et al. Occurrence and frequency of arrhythmias in hypertrophic cardiomyopathy in relation to delayed enhancement on cardiovascular magnetic resonance. J Am Coll Cardiol 2008;51:1369-74.

15. Maron MS, Appelbaum E, Harrigan $\mathrm{CJ}$, et al. Clinical profile and significance of delayed enhancement in hypertrophic cardiomyopathy. Circ Heart Fail 2008;1: 184-91.

16. Rudolph A, Abdel-Aty H, Bohl S, et al. Noninvasive detection of fibrosis applying contrast-enhanced cardiac magnetic resonance in different forms of left ventricular hypertrophy relation to remodeling. J Am Coll Cardiol 2009;53:284-91.

17. Germans T, Wilde AA, Dijkmans PA, et al. Structural abnormalities of the inferoseptal left ventricular wall detected by cardiac magnetic resonance imaging in carriers of hypertrophic cardiomyopathy mutations. J Am Coll Cardiol 2006;48: 2518-23.

18. Moon JC, Mogensen J, Elliott PM, et al. Myocardial late gadolinium enhancement cardiovascular magnetic resonance in hypertrophic cardiomyopathy caused by mutations in troponin I. Heart 2005;91: 1036-40.

19. Crilley JG, Boehm EA, Blair E, et al. Hypertrophic cardiomyopathy due to sarcomeric gene mutations is characterized by impaired energy metabolism irrespective of the degree of hypertrophy. J Am Coll Cardiol 2003;41:1776-82.

20. Debold EP, Schmitt JP, Patlak JB, et al. Hypertrophic and dilated cardiomyopathy mutations differentially affect the molecular force generation of mouse alphacardiac myosin in the laser trap assay. Am J Physiol Heart Circ Physiol 2007;293: H284-H291.

21. Lombardi R, Rodriguez G, Chen SN, et al. Resolution of established cardiac hypertrophy and fibrosis and prevention of systolic dysfunction in a transgenic rabbit model of human cardiomyopathy through thiol-sensitive mechanisms. Circulation 2009;119:1398-407.

22. Tardiff JC. Sarcomeric proteins and familial hypertrophic cardiomyopathy: linking mutations in structural proteins to complex cardiovascular phenotypes. Heart Fail Rev 2005;10:237-48.

23. Camici PG, Crea F. Coronary microvascular dysfunction. N Engl J Med 2007; 356:830-40.

24. Geisterfer-Lowrance AA, Christe M, Conner DA, et al. A mouse model of familial hypertrophic cardiomyopathy. Science 1996;272:731-4.

25. Kim JB, Porreca GJ, Song L, et al. Po- lony multiplex analysis of gene expression (PMAGE) in mouse hypertrophic cardiomyopathy. Science 2007;316:1481-4.

26. González A, López B, Ravassa S, et al. Biochemical markers of myocardial remodelling in hypertensive heart disease. Cardiovasc Res 2009;81:509-18.

27. Querejeta R, López B, González A, et al. Increased collagen type I synthesis in patients with heart failure of hypertensive origin: relation to myocardial fibrosis. Circulation 2004;110:1263-8.

28. Klappacher G, Franzen P, Haab D, et al. Measuring extracellular matrix turnover in the serum of patients with idiopathic or ischemic dilated cardiomyopathy and impact on diagnosis and prognosis. Am J Cardiol 1995;75:913-8.

29. Poulsen SH, Host NB, Egstrup K. Long-term changes in collagen formation expressed by serum carboxyterminal propeptide of type-I procollagen and relation to left ventricular function after acute myocardial infarction. Cardiology 2001; 96:45-50.

30. Zannad F, Alla F, Dousset B, Perez A, Pitt B. Limitation of excessive extracellular matrix turnover may contribute to survival benefit of spironolactone therapy in patients with congestive heart failure: insights from the Randomized Aldactone Evaluation Study (RALES). Circulation 2000;102:2700-6.

31. Lombardi R, Betocchi S, Losi MA, et al. Myocardial collagen turnover in hypertrophic cardiomyopathy. Circulation 2003; 108:1455-60.

32. Fassbach M, Schwartzkopff B. Elevated serum markers for collagen synthesis in patients with hypertrophic cardiomyopathy and diastolic dysfunction. Z Kardiol 2005;94:328-35.

33. Roldán V, Marín F, Gimeno JR, et al. Matrix metalloproteinases and tissue remodeling in hypertrophic cardiomyopathy. Am Heart J 2008;156:85-91.

34. Noji Y, Shimizu M, Ino H, et al. Increased circulating matrix metalloproteinase-2 in patients with hypertrophic cardiomyopathy with systolic dysfunction. Circ J 2004;68:355-60.

35. Sluysmans T, Colan SD. Theoretical and empirical derivation of cardiovascular allometric relationships in children. J Appl Physiol 2005;99:445-57.

36. McKenna WJ, Spirito P, Desnos M, Dubourg O, Komajda M. Experience from clinical genetics in hypertrophic cardiomyopathy: proposal for new diagnostic criteria in adult members of affected families. Heart 1997;77:130-2.

37. Lang RM, Bierig M, Devereux RB, et al. Recommendations for chamber quantification: a report from the American Society of Echocardiography's Guidelines and Standards Committee and the Chamber Quantification Writing Group, developed in conjunction with the European Association of Echocardiography, a branch of the European Society of Cardiology. J Am Soc Echocardiogr 2005;18: 1440-63.

38. Ommen SR, Nishimura RA, Appleton $\mathrm{CP}$, et al. Clinical utility of Doppler echocardiography and tissue Doppler imaging in the estimation of left ventricular filling pressures: a comparative simultaneous Doppler-catheterization study. Circulation 2000;102:1788-94.

39. Marino P, Little WC, Rossi A, et al. Can left ventricular diastolic stiffness be measured noninvasively? J Am Soc Echocardiogr 2002;15:935-43.

40. Nagueh SF, Shah G, Wu Y, et al. Altered titin expression, myocardial stiffness, and left ventricular function in patients with dilated cardiomyopathy. Circulation 2004;110:155-62.

41. Grothues F, Smith GC, Moon JC, et al. Comparison of interstudy reproducibility of cardiovascular magnetic resonance with two-dimensional echocardiography in normal subjects and in patients with heart failure or left ventricular hypertrophy. Am J Cardiol 2002;90:29-34.

42. Kim RJ, Fieno DS, Parrish TB, et al. Relationship of MRI delayed contrast enhancement to irreversible injury, infarct age, and contractile function. Circulation 1999;100:1992-2002.

43. Trivedi P, Risteli J, Risteli L, Hindmarsh PC, Brook CG, Mowat AP. Serum concentrations of the type I and III procollagen propeptides as biochemical markers of growth velocity in healthy infants and children and in children with growth disorders. Pediatr Res 1991;30:276-80.

44. López B, González A, Díez J. Circulating biomarkers of collagen metabolism in cardiac diseases. Circulation 2010;121: 1645-54.

45. Wu E, Judd RM, Vargas JD, Klocke FJ, Bonow RO, Kim RJ. Visualisation of presence, location, and transmural extent of healed Q-wave and non-Q-wave myocardial infarction. Lancet 2001;357:21-8.

46. Ricciardi MJ, Wu E, Davidson CJ, et al. Visualization of discrete microinfarction after percutaneous coronary intervention associated with mild creatine kinase-MB elevation. Circulation 2001;103:2780-3.

47. Strauss DG, Selvester RH, Lima JA, et al. ECG quantification of myocardial scar in cardiomyopathy patients with or without conduction defects: correlation with cardiac magnetic resonance and arrhythmogenesis. Circ Arrhythm Electrophysiol 2008;1:327-36.

48. Niimura H, Bachinski LL, Sangwatanaroj $\mathrm{S}$, et al. Mutations in the gene for cardiac myosin-binding protein $\mathrm{C}$ and late-onset familial hypertrophic cardiomyopathy. N Engl J Med 1998;338:1248-57.

49. Niimura H, Patton KK, McKenna WJ, et al. Sarcomere protein gene mutations in hypertrophic cardiomyopathy of the elderly. Circulation 2002;105:446-51.

Copyright (C) 2010 Massachusetts Medical Society. 